Please cite as: A. Akbari, A. Akbarzadeh-Sharbaf and D. D. Giannacopoulos, "Finite-Element Gaussian Belief Propagation Solver for Multi-Physics Modeling of Radiofrequency Tumor Ablation," in IEEE Transactions on Magnetics, vol. 55, no. 6, pp. 1-4, June 2019, Art no. 7201604, doi: 10.1109/TMAG.2019.2896583.

\title{
Finite Element Gaussian Belief Propagation Solver for Multi-Physics Modeling of Radiofrequency Tumor Ablation
}

\author{
Amir Akbari, Ali Akbarzadeh-Sharbaf, Student Member, IEEE, and Dennis D. Giannacopoulos, Senior Member, IEEE \\ Department of Electrical and Computer Engineering, McGill University, Montreal, QC H3A 0E9, Canada
}

\begin{abstract}
A new finite element Gaussian belief propagation (FGaBP) algorithm for multi-physics applications is presented in this paper. The FGaBP method is first modified for solving the heat-transfer problem and then verified against an analytical solution. Combined with a Gauss-Seidel algorithm, FGaBP is used to solve the coupled electrical-thermal problem that emerges in radiofrequency ablation of hepatic tumors while the extent of ablated zone is computed for a simplified model. The multi-physics FGaBP algorithm preserves the parallel scalability of FGaBP as in previous uni-physics works.
\end{abstract}

Index Terms-FGaBP, Gauss-Seidel algorithm, Radiofrequency Ablation (RFA).

\section{INTRODUCTION}

$\mathbf{T}$ HE finite element Gaussian belief propagation (FGaBP) method [1] is an alternative to the traditional finiteelement formulation, whose main advantage is providing abundant parallelism based on localized computations and distributed message communication. While FGaBP uses message passing for communications between different parts of the domain, the same approach could be utilized for communication between different physics in a multi-physics scheme. This work presents a multi-physics reformulation of FGaBP realized by combining its localized computations and message passing scheme with the coupled phenomena that appear in radiofrequency ablation (RFA) of hepatic tumors.

RFA is a technique that is employed in destruction of tumors in different locations including liver, kidney, lung, bone, prostate, and breast. It uses devices operating between $460-550 \mathrm{kHz}$ delivering electrical currents to biological tissues to thermally damage a tumor by raising its temperature to approximately $100^{\circ} \mathrm{C}$ for a period of $10-15$ minutes. Effective RFA is defined as an entire ablation of the tumor with a safety margin of destroyed healthy tissue in its vicinity, which in turn relies on optimal probe placements and the extent of ablation zone. However for treating tumors in the liver, the latter is difficult to control as the hepatic blood vessels and the parenchyma perfusion dissipate heat and change the size and shape of the lesion zone. In fact, clinical evidence shows a noteworthy mismatch between expected and observed ablation zone leading to reduced survival rates due to over-treatment with severe injuries (up to $9 \%$ ) or under-treatment with tumor recurrence (up to $40 \%$ ) [2].

Theoretical models and computer simulations are noninvasive powerful tools providing critical information on the electrical and thermal behavior of RFA. Recently, computational models of RFA have gained attention as a tool for studying the heat distribution around the ablation probes [3], [4]. In order to simulate RFA therapies and predict the extent of the ablation zone, a multi-physics approach that combines electrical-thermal heating process with a biophysical model of

Manuscript received November 4, 2018. Corresponding author: A. Akbari (email: amir.akbari@mail.mcgill.ca). the heat transfer and cellular necrosis is needed. To investigate the capabilities of FGaBP for solving multi-physics problems, the coupled electrical-thermal phenomenon in a simplified model of RFA is simulated in this paper. Section II presents the mathematical model of electrical heating, heat transfer and their coupling in the liver. Section III provides a review on the FGaBP algorithm and introduces a new multi-physics FGaBP method, based on a Gauss-Seidel algorithm; Section IV describes a test case and the results obtained.

\section{Modeling of Electrical Heating And Heat TRANSFER IN THE LIVER}

Modeling of RFA is based on a transient analysis of a coupled electrical-thermal problem. The spatial distribution of temperature inside the tissues is obtained by solving the bioheat equation [5]:

$$
\rho c_{t i} \frac{\partial T}{\partial t}=\nabla \cdot(d \nabla T)+Q
$$

where $\rho$ is the mass density $\left(\mathrm{kg} / \mathrm{m}^{3}\right), c_{t i}$ is the tissue specific heat $\left(\mathrm{J}(\mathrm{kgK})^{-1}\right), T$ is the temperature $\left({ }^{\circ} \mathrm{C}\right), d$ is the thermal conductivity $\left(\mathrm{W}(\mathrm{mK})^{-1}\right)$, and $Q$ is the heat source $\left(\mathrm{W} / \mathrm{m}^{3}\right)$. We add the cooling term $H\left(T_{b o}-T\right) /(1-\varepsilon)$ to (1) when a point belongs to a large liver vessel or $-\varepsilon \rho c_{b l} \mathbf{v} \cdot \nabla T /((1-\varepsilon)$ when it belongs to the parenchyma. Here, $c_{b l}$ is the blood specific heat, $\mathbf{v}$ and $\varepsilon$ stand for blood velocity and blood volume fraction (fraction of blood volume over total volume), and $T_{b o}$ is the normal body temperature, i.e $37^{\circ} \mathrm{C}$. At the frequencies employed in RFA $(460-550 \mathrm{kHz})$ the displacement currents are negligible and the electrical problem is resolved using Laplace's equation:

$$
\nabla \cdot(\sigma(T) \nabla v)=0
$$

where $\nabla$ is the gradient operator, $\sigma(T)$ is the temperaturedependent electrical conductivity $(\mathrm{S} / \mathrm{m})$, and $v$ is the electric potential $(\mathrm{V})$. The bio-heat equation is coupled to the Laplacian equation by its source term $Q=\sigma|\nabla v|^{2}$. On the other hand, most RFA models consider a temperaturedependent change in $\sigma$ using a temperature coefficient of $+2 \% /{ }^{\circ} \mathrm{C}$ [3]; Thus, the thermal profile and the electric 


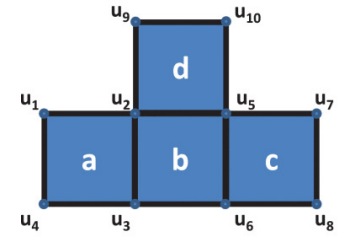

(a)

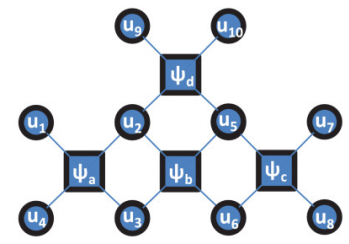

(b)
Fig. 1. Example quadrilateral (1st order) (a) FEM mesh and (b) its equivalent factor graph [6].

field are interdependent. After using theta-scheme for timediscretization of the heat transient problem, and similar space discretization for both (1) and (2), we will have the following set of coupled discrete equations:

$$
\begin{gathered}
{\left[M_{v}(T)\right]\{v\}=0} \\
{\left[M_{T}+\delta t \theta S_{T}\right]\{T\}^{n+1}=\left[M_{T}-\delta t(1-\theta) S_{T}\right]\{T\}^{n}+} \\
\delta t\left\{(1-\theta)\{f(v)\}^{n}+\theta\{f(v)\}^{n+1}\right\}
\end{gathered}
$$

where $M_{v, i j}(T)=\int \sigma(T) N_{i} N_{j} d V, M_{T, i j}=\rho c \int N_{i} N_{j} d V$, $S_{T, i j}=d \int \vec{\nabla} N_{i} \cdot \vec{\nabla} N_{i} d V$ and $f_{j}(v)=\int \sigma(T) N_{j}|\nabla v|^{2} d V$. The choice of solution approach for these coupled equations depends on a number of considerations. From a practical standpoint, existing codes for component solutions motivates successive substitution as the route to a first multi-physics simulation, which is typically done in a Gauss-Seidel iterative manner. A conventional Gauss-Seidel algorithm solves the uniphysics problems sequentially, however, as explained in the next section, the message passing scheme of FGaBP and its localized computations allow us to solve the coupled electrical and thermal equations in parallel in order to make a more efficient use of the processing resources.

\section{SOLVING THE COUPLED PROBLEM WITH FGABP}

FGaBP reformulates FEM into an inference problem over a factor graph, which may then be solved applying the belief propagation rules. As shown in Fig. 1, there are two distinct nodes in the factor graph, variable nodes ( $\mathrm{VN}$, nodes of unknowns) represented by circles, and factor nodes (FNs) represented by squares. FGaBP assumes the solution at each $\mathrm{VN}$ is a random variable with a Gaussian distribution whose shape is defined by two parameters, $\alpha$ and $\beta$, where $\alpha$ is the reciprocal of the variance and $\beta / \alpha$ is the mean. By passing two types of messages, factor node messages and variable node messages, FGaBP tries to find the values of $\alpha$ and $\beta$ for each node. A factor node message, $m_{a i}$, is sent from factor node $a$ $\left(\mathrm{FN}_{\mathrm{a}}\right)$ to the connected variable node $i\left(\mathrm{VN}_{\mathrm{i}}\right)$ and represents the most probable state of $u_{i}$, as observed from $\mathrm{FN}_{\mathrm{a}}$. In return, the variable node message $\eta_{i a}$ is a distribution in terms of $u_{i}$ representing observations from other connected FNs.

The main steps applied in FGaBP are as follows:

1) The first step updates $\beta$ and $\alpha$ values for all VNs associated with each FN. This operation is done by each FN sending messages to all connected VNs, which then updates the local $\alpha$ and $\beta$ values. This requires solving two small systems of equations [6]:

$$
\begin{aligned}
& \alpha_{a i}^{t}=M_{L(i)}-V^{T}\left(\bar{W}^{\left(t_{*}\right)}\right) V \\
& \beta_{a i}^{t}=B_{L(i)}-\left(\bar{K}^{\left(t_{*}\right)}\right)^{T}\left(\bar{W}^{\left(t_{*}\right)}\right)^{-1} V .
\end{aligned}
$$

Noteworthy, $M, V, W$, and $K$ are small matrices corresponding to the local factors $\psi_{a}$ and $t_{*}$ represents the previous value.

2) In the second step, each $\mathrm{VN}$ receives the new beliefs from the connected FNs, computes VN messages accordingly and sends them back to each neighboring FN [6]:

$$
\begin{aligned}
\alpha_{i}^{(t)} & =\sum_{k \in N(i)} \alpha_{k i}^{\left(t_{*}\right)}, \alpha_{i a}^{(t)}=\alpha_{i}^{(t)}-\alpha_{a i}^{\left(t_{*}\right)} \\
\beta_{i}^{(t)} & =\sum_{k \in N(i)} \beta_{k i}^{\left(t_{*}\right)}, \beta_{i a}^{(t)}=\beta_{i}^{(t)}-\beta_{a i}^{\left(t_{*}\right)}
\end{aligned}
$$

where $N(i)$ is the set of all FNs connected to $\mathrm{VN}_{\mathrm{i}}$.

These steps are repeated until the changes in $\alpha$ and $\beta$ values reach a certain threshold. After convergence, the solution is recovered computing the median as $\mu=\beta / \alpha$. It is important to observe that FNs (where most of the computation is done) are only connected through variable nodes and that messages are only sent to local neighboring FNs. This is a key feature that gives FGaBP great potential for parallel processing; in this paper, we show that this parallelism of FGaBP could be preserved in a multi-physics scheme.

In order to solve the coupled electrical-thermal problem described in Section II with FGaBP, we should have messages $\alpha_{v}$ and $\beta_{v}$ in the electrical problem and $\alpha_{T}$ and $\beta_{T}$ in the thermal problem. We can exploit the local computations and message passing strategies used in $\mathrm{FGaBP}$ in order to derive a parallel Gauss-Seidel algorithm. Unlike a classical Gauss-Seidel in which the uni-physics problems are solved sequentially, in the proposed parallel algorithm we can solve both electrical and thermal problems in parallel. This method is shown in Algorithm 1. The electrical problem updates its local FN matrices according to the temperature obtained in the previous iteration (or the initial temperature for the first iteration) and starts calculating $\beta_{v}$ and $\alpha_{v}$ messages for each cell. A local convergence could be satisfied if the messages of one cell or one group of cells are converged. The electrical component sends the updated $\beta_{v}$ and $\alpha_{v}$ messages of the locally converged cells to the thermal component. The thermal problem then updates the local right hand side (RHS) matrices of the corresponding cells and starts calculating $\beta_{T}$ and $\alpha_{T}$ messages while waiting for more cells in the electrical problem to be converged. Whenever a number of cells are converged in the thermal problem, the FN matrices of those cells are updated accordingly in the electrical problem and messages are calculated again. This procedure continues until a convergence criterion such as L2-norm of the electrical conductivity is smaller than a threshold. To make FGaBP solver converge faster, we can use the values of $\alpha$ and $\beta$ messages from the previous iteration. Hence, the messages $\alpha_{v}$ and $\beta_{v}$ in the electrical problem and $\alpha_{T}$ and $\beta_{T}$ in the thermal problem 
only need to be initialized in the first iteration and then will continue by just updating their values. In fact, as shown by [6], we only need to update $\beta_{v}$ and $\beta_{T}$ after a few iterations since $\alpha$ messages converge very fast.

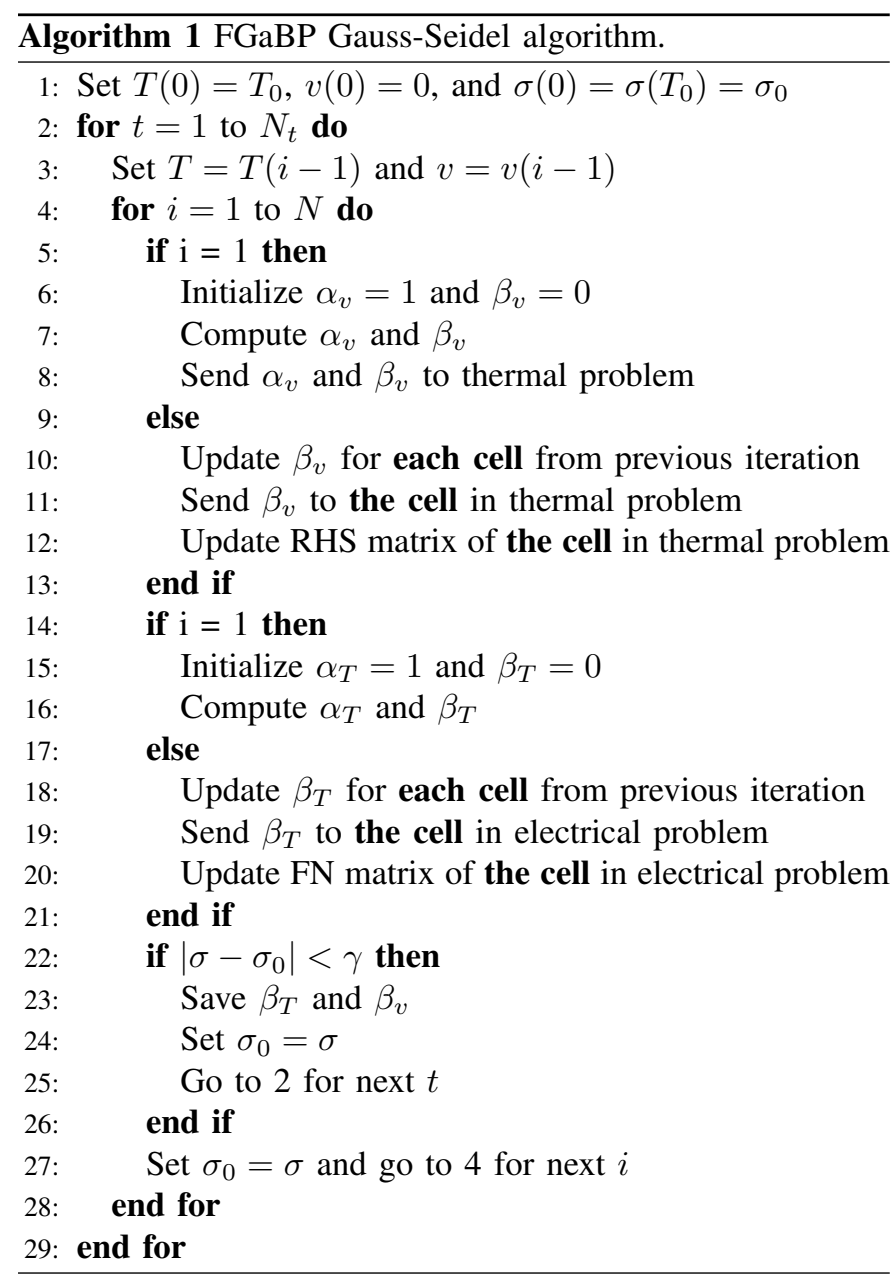

\section{NuMERICAL RESUlTS}

The tests were performed on an Intel Core i7-5960X CPU clocked at $3 \mathrm{GHz}$, with eight cores (totaling 16-threads) and 16 GB DDRAM3. The operating system installed was 64b Ubuntu Linux 14.04.4 LTS (kernel 3.13.0-79), with GNU Compiler Collection version 4.9.3. Also, the code was developed using the deal.II 8.2.1 library.

\section{A. Quantitative Verification of the Heat Transfer Model}

To evaluate the FGaBP heat transfer solver, its behavior is compared on a regular rectangular domain with an analytical solution. For a source released at $x_{0}$ at time $t_{0}$, the 2D analytical solution of the diffusion equation: $\rho c_{t i}(\partial T / \partial t)=$ $\nabla \cdot(d \nabla T)+Q$ is [7]:

$$
T(x, t)=\frac{M}{\left[4 \pi\left(t-t_{0}\right) D\right]} \exp \left(\frac{-\left\|x-x_{0}\right\|^{2}}{4 D\left(t-t_{0}\right)}\right)
$$

To have the same conditions as the RFA computation on a patient's liver, parameters $\rho, c_{t i}$ and $d$ have the values $1.06 \times 10^{3} \mathrm{~kg} / \mathrm{m}^{3}, 3.06 \times 10^{3} \mathrm{~J}(\mathrm{kgK})^{-1}$, and $0.512 \mathrm{~W}(\mathrm{mK})^{-1}$ as reported for liver in the literature [5].
We initialized the temperature values at each point of the domain with the analytical solution at time $t=0$ with this set of parameters: $M=450{ }^{\circ} \mathrm{C} \cdot \mathrm{mm}^{2}, D=0.15 \mathrm{~mm}^{2} / \mathrm{s}$, $t_{0}=-0.1 \mathrm{~s}, x_{0}=(0,0)$. The diffusion equation is solved using our FGaBP solver and the temperature at a typical point of the domain is reported and compared with the analytical solution values. Neumann boundary conditions were used at the border of the domain, which was chosen to be large enough to get rid of the boundary effect at the probed points.

Since the theta-scheme with $\theta=0.5$ is used for time discretization, no hard restrictions for time-step exist, but in practice we still want to make the time-step smaller to decrease the error. In the FGaBP heat transfer simulation a time-step of $0.2 \mathrm{~s}$ appeared to be a good compromise between accuracy and computational time. When the time-step is fixed to a constant value, a non-oscillatory criteria imposes an upper bound for the spatial resolution as [8] :

$$
\Delta x^{2} \leq \frac{20 \theta d}{\rho c_{t i}} \Delta t
$$

This means for $\Delta t=0.2 \mathrm{~s}, \theta=0.5$, and with other parameters values as in the literature [5], $\Delta x$ must be smaller than $0.53 \mathrm{~mm}$. In Fig. 2 the analytical solution and FGaBP solution with a time-step of $0.2 \mathrm{~s}$ and different spatial resolutions are plotted. Fig. 2 shows the smaller the spatial resolution, the closer the computed solution is to the analytical one. Quantitatively, the Root-Mean-Square (RMS) errors between the computed solution and the analytical one decreased with the resolution: $3.31{ }^{\circ} \mathrm{C}, 0.5{ }^{\circ} \mathrm{C}, 0.14{ }^{\circ} \mathrm{C}$ for $0.625 \mathrm{~mm}, 0.5 \mathrm{~mm}$ and $0.2 \mathrm{~mm}$, respectively. This analysis confirms qualitatively and quantitatively the accuracy of the implementation of the heat transfer model with FGaBP.

\section{B. Coupled Electrical-Thermal Computation}

In order to simulate the FGaBP Gauss-Seidel algorithm, we used the same geometry, mesh size, time-step and parameter values as in the heat transfer model. As shown in Fig. 3, left, the conducting tip of the electrode is embedded into a $8.0 \mathrm{~cm}$ by $8.0 \mathrm{~cm}$ rectangular region that simulates tissue surrounding the probe tip. A source voltage of $16 \mathrm{~V}$ is applied to the conducting tip of the probe. All of the outer boundaries of the rectangular domain serve as a return ground electrode. Regarding the thermal problem, the initial value of the temperature is chosen to be the normal body temperature, i.e. $37^{\circ} \mathrm{C}$ and a Dirichlet boundary condition of $37^{\circ} \mathrm{C}$ is applied at the outer boundary.

The temperature distribution obtained from the FGaBP Gauss-Seidel algorithm is depicted in Fig. 3, right. To verify the correctness of the algorithm, the computed temperature at a certain point in the domain is compared to that obtained from the FGaBP when used in conventional Gauss-Seidel iterations. Fig. 4 shows the transient temperature computed from both methods; the RMS error between them is less than $0.01{ }^{\circ} \mathrm{C}$ which confirms the correctness of our algorithm.

\section{Computational Efficiency}

In Section III, we merged a Gauss-Seidel algorithm into FGaBP iterations and designed a new algorithm. We claimed that this approach is more efficient than using FGaBP in a 


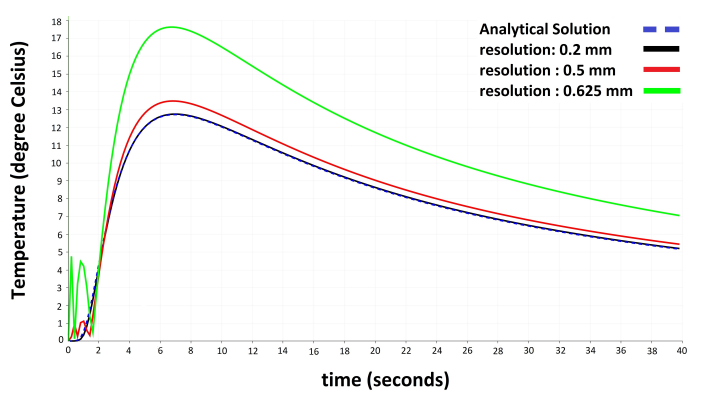

Fig. 2. Temperature distribution for an instantaneous point source using different space resolutions and time-step $=0.2 \mathrm{~s}$ (Colored figure provided in the electronic version).
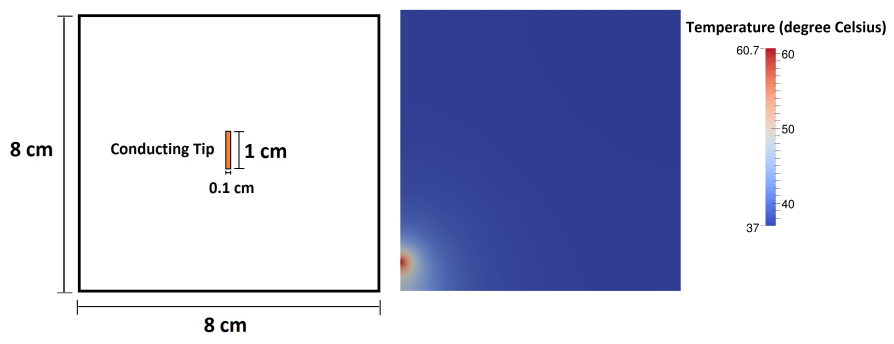

Fig. 3. (Left): The geometry of the simple test case. (Right): The coupled problem temperature distribution in the upper right quadrant of the domain from the multi-physics FGaBP algorithm.

classical Gauss-Seidel since it makes FGaBP solver converge faster. This can be confirmed by using FGaBP in a classical Gauss-Seidel to obtain voltage and temperature values at each iteration, and then comparing the total number of FGaBP iterations with that obtained from Algorithm 1. The results indicate that with similar convergence criterion, although Algorithm 1 needs more Gauss-Seidel iterations to converge, its total number of FGaBP iterations is about half of that needed in the conventional Gauss-Seidel. To test the parallel scalability properties of Algorithm 1, a CPU implementation with multithreading (OpenMP) is provided. For this purpose, the grid was further refined to a medium size problem with $1,000,000$ elements. The performance scales up almost linearly up to six threads, experiencing some degradation when the number of threads reaches eight which is the number of physical cores, probably because the CPU is also used for processing instructions of the operating system. The performance is improved again when the number of threads is further increased up to fourteen threads. The results show speedups of more than six times with respect to one CPU core.

\section{CONCLUSION}

A novel method combining the FGaBP formulation and Gauss-Seidel iterations has been proposed to solve the electrical-thermal coupled problem in RFA of hepatic tumors. First, the time-domain heat-transfer equation with thetascheme time discretization is solved by FGaBP and verified against an analytical solution; then, the coupled electricalthermal problem is solved with the new FGaBP Gauss-Seidel algorithm for a simple test case. In order to verify the correctness of the algorithm, obtained results are compared

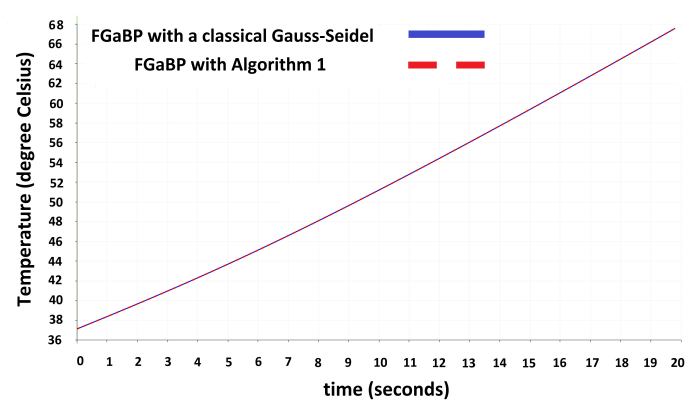

Fig. 4. Temperature distribution for the coupled problem obtained from the multi-physics FGaBP algorithm and a classical Gauss-Seidel algorithm (Colored figure provided in the electronic version).

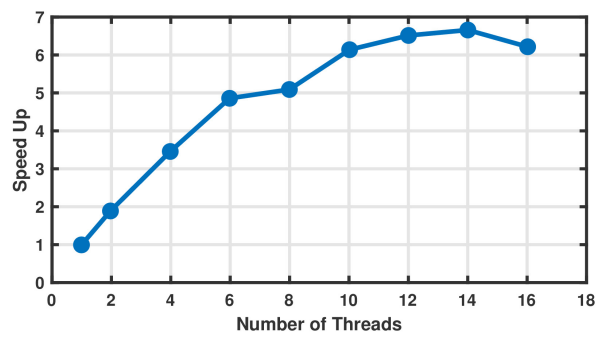

Fig. 5. Performance scaling of the multi-physics FGaBP method in terms of speedup with respect to 1 core implementation.

with the classical Gauss-Seidel, showing good fidelity. The parallel scalability of the FGaBP method is retained in the new multi-physics version proposed here. Our results show speedups of more than six times with respect to one CPU core. Extending the multi-physics FGaBP algorithm to more complicated modelings of RFA, including a complete coupled electrical-thermal-cellular necrosis problem in the liver with a complex geometry will be addressed in future work.

\section{REFERENCES}

[1] Y. El-Kurdi, D. Giannacopoulos, and W. J. Gross, "Relaxed gaussian belief propagation," in Information Theory Proceedings (ISIT), 2012 IEEE International Symposium on. IEEE, 2012, pp. 2002-2006.

[2] S. L. Wong, P. B. Mangu, M. A. Choti, T. S. Crocenzi, G. D. Dodd III, G. S. Dorfman, C. Eng, Y. Fong, A. F. Giusti, D. Lu et al., "American society of clinical oncology 2009 clinical evidence review on radiofrequency ablation of hepatic metastases from colorectal cancer," Journal of Clinical Oncology, vol. 28, no. 3, pp. 493-508, 2009.

[3] E. J. Berjano, "Theoretical modeling for radiofrequency ablation: stateof-the-art and challenges for the future," Biomedical engineering online, vol. 5, no. 1, p. 24, 2006.

[4] V. D'Ambrosio, P. Di Barba, F. Dughiero, M. Mognaschi, and A. Savini, "Non-invasive thermometry for the thermal ablation of liver tumor: A computational methodology," International Journal of Applied Electromagnetics and Mechanics, vol. 25, no. 1-4, pp. 407-412, 2007.

[5] C. Audigier, T. Mansi, H. Delingette, S. Rapaka, V. Mihalef, D. Carnegie, E. Boctor, M. Choti, A. Kamen, N. Ayache et al., "Efficient lattice boltzmann solver for patient-specific radiofrequency ablation of hepatic tumors," IEEE Transactions on Medical Imaging, vol. 34, no. 7, pp. 15761589,2015

[6] Y. El-Kurdi, M. M. Dehnavi, W. J. Gross, and D. Giannacopoulos, "Parallel finite element technique using gaussian belief propagation," Computer Physics Communications, vol. 193, pp. 38-48, 2015.

[7] E. Kersal, "Analytic solutions of partial differential equations," University of Leeds, Leeds, 2004.

[8] W. Cui, K. Gawecka, D. Taborda, D. Potts, and L. Zdravković, "Timestep constraints in transient coupled finite element analysis," International Journal for Numerical Methods in Engineering, vol. 106, no. 12, pp. $953-$ 971, 2016. 